\title{
Cost and Return Analysis of Beneficiaries and Non-Beneficiary Farm Pond Farmers for Soybean Cultivation in Washim District
}

\author{
A. D. Chakranarayan*, S. C. Nagpure, J. R. Kankate and R. K. Patil \\ Department of Agriculture Economics and Statistics, PGI, \\ Dr. PDKV Akola, (MH) 444 104, India \\ *Corresponding author
}

\section{Keywords}

Soybean,

Profitability,

Output-input ratio,

Productivity, Cost

and returns, Input

\section{Article Info}

Accepted:

16 November 2020

Available Online:

10 December 2020

\begin{abstract}
A B S T R A C T
The present study entitled, 'Cost and return analysis of beneficiaries and non-beneficiary farm pond farmers for soybean cultivation in Washim District. The study was undertaken to examine the impact of farm ponds on production of soybean crops. There were 50 beneficiary farmers having farm ponds and 50 non-beneficiary farmers without farm ponds on their field were selected from Malegaon tahasil of Washim district. 10 villages from Malegaon tahasil were selected purposively and, from each village sufficient samples of beneficiary and non-beneficiary farmers were taken randomly for comparison. The selected farmers were classified into three categories viz., small, medium, large according to their land holding. The primary data was collected from the farmers by survey method and cost concept i.e., cost 'A', cost ' $\mathrm{B}$ ' and cost ' $\mathrm{C}$ ' was used for the analysis of data. The beneficiary and non-beneficiary farmers per hectare cost of cultivation of soybean crop for the sample as an overall was Rs. 89555.54 per ha and Rs. 75716.89 per ha. respectively. It is observed from the study that in case of beneficiary farmers at overall level the Outputinput ratio at cost ' $\mathrm{C}$ ' was 1.59 , while in case of non-beneficiary farmers it was 1.38 . It shows that the beneficiary farmers were more profitable than non-beneficiary farmers.
\end{abstract}

\section{Introduction}

Indian economy is predominantly rural and agriculture oriented where the declining trend in the average size of the farm holding poses a serious problem. In agriculture 84 percent of the holding is less than 2 acres. Majority of them are dry lands and even irrigated areas depend on the vagaries of monsoon. In this context, if farmers concentrated on crop production they will be subjected to a high degree of uncertainty in income and employment. Hence, it is imperative to evolve suitable strategy for augmenting the income of the small and marginal farmers by combining to increase the productivity and supplement the income. In an agricultural country like India, the average land holding is very small. Moreover, the economy is mainly dependent on stability of crop production in rainfed areas. Construction of farm ponds is one of the beneficial programmes for harvesting excess rain water during rainy season; which is implemented by the State 
Agricultural Development under National Agricultural Development Programme (Rashtriya Krishi Vikas Yojana) etc. Water is an essential and precious resource upon which our ecosystems and agricultural production depend. However, water a natural resource of the world constitutes, 1,384 million cubic kilometers of which around 97.39 per cent (i.e. 1,348 million cubic kilometers) of water is in the oceans, which is salty in nature. Another 2.61 per cent (i.e. 36 million $\mathrm{km} 3$ ) is fresh water of this 77.23 per cent $(27.82$ million $\mathrm{km} 3$ ) is in the polar ice caps, icebergs and glaciers. Only small fraction of water resources ( 0.59 per cent or 8.2 million $\mathrm{km} 3)$ of the earth present on the ground, lakes, rivers and atmosphere and is useful to mankind. Whereas, more than 99 per cent of water present on the earth is not useful to mankind.

Farm ponds are created in various states of India along with Maharashtra. The main aim of constructing farm pond is to make the availability of protective irrigation at critical growth stages of crop. In Maharashtra through various scheme of government the farm ponds are allotted to farmers namely, National Horticultural Mission, Mahatma Gandhi National Rural Employment Guarantee Act (MGNREGA) and Mahatma Phule Jal Abhiyan etc. The farm pond has a great impact on changing the crop productivity as well as cropping intensity. It also helps in changing the economic situation of farmers. The irrigated area also increases due to the construction of farm pond (Deshmukh and Hyalij, 2017).

The rainfall amount and its distribution during monsoon period are mostly erratic and uncertain coupled with occurrence of frequent droughts of several days to weeks affecting rainfed productivity drastically. A farm pond is a large hole dug out in the earth, usually square or rectangular in shape, which harvest rainwater and stores it for future use. It has inlet to regulate inflow and an outlet to discharge excess water. The pond is surrounded by a small bund, which prevents erosion on the banks of pond. The size and depth depend on the amount of land available, the type of soil, the farmers water requirements, the cost of excavation, and the possible uses of the excavated earth. Water from the pond is conveyed to the fields manually, by pumping or by both methods. Farm pond size adopted by the farmers ranges $15 \times 15 \times 3$ meter, $20 \times 20 \times 3$ meter, $25 \times 25 \times 3$ meter, and $30 \times 30 \times 3$ meter according to size of land holding of a farmer. (Mane N. P., S. S. Thakre, 2015).

\section{Materials and Methods}

The present study was undertaken with main object of determining inputs used, cost, returns, profitability and resource use efficiency from soybean production. The share of each item to the total cost i.e. cost ' $C$ ' total economic costs for soybean cultivation. The cost has determined on the basis of standard cost concept i.e. cost 'A', cost 'B', cost ' $\mathrm{C}$ ', the different cost concepts have different utilities in research.

\section{$\operatorname{Cost}^{6} \mathrm{~A}$ '}

1. Hired human labour

2. Bullock labour

3. Machinery hours

4. Seed

5. Irrigation charges

6. Land revenue and other cases

7. Depreciation

8. Interest on working capital

\section{Cost ' $B$ '}

Cost ' $\mathrm{B}$ ' was estimated by adding interest on fixed capital and rental value of land to Cost 'A' i.e. 
Cost ' $\mathrm{B}$ ' = Cost ' $\mathrm{A}$ ' + Rental value of owned land + Interest on owned fixed capital (excluding land) @ 10\%

\section{Cost ' $C$ '}

It is obtained by adding imputed value of family labour to cost ' $\mathrm{B}$ '

Cost ' $\mathrm{C}$ ' $=$ Cost ' $\mathrm{B}$ ' + Imputed value of family labour

Gross income: It is calculated as under,

Gross value of output $=$ value of main produce + value of by-produce

\section{Net income}

\section{Gross value of output - Cost ' $C$ '}

Input-output ratio: It is ratio between the value of gross output and the cost of cultivation at different cost concept.

\section{Results and Discussion}

\section{Per hectare input utilization for Soybean}

The degree of management of the resources can be judged for the utilization of resources, the choice and the decision making. Beside this, it is also indicating the level of technology adopted by the farmers. Results revealed that in table 1 indicate that at overall level the use of various inputs for soybean on beneficiary farms was slightly higher than non-beneficiary farms. Use of seed and farm yard manure per hectare did not vary much among the beneficiary and non-beneficiary farms. Use of chemical fertilizers on beneficiary farms was slightly higher than the non-beneficiary farms. Intergroup comparison of beneficiary and non-beneficiary farms also revealed the same trend.

\section{Per hectare cost of cultivation for Soybean}

For the purpose the total variable cost, total production, gross return and net return were worked out separately for each crop. Table 2 revealed that, the beneficiary and nonbeneficiary farmers per hectare cost of cultivation of soybean crop for the sample as an overall was per ha. total cost of cultivation i.e. cost ' $C$ ' ranges from Rs.89555.54 in large size group it was Rs.246691.6 in medium size group it was Rs.58106.79 and in case of small size group of farmers it was Rs. 55217.24 respectively. The per ha. cost of cultivation for Large farmers was Rs.89555.54 Higher total cost on large size farmer was obviously due to higher use of input.

In non-beneficiary group costs were lower as compared to beneficiary farmers as there was low level of input used. In beneficiary group the important items of expenditure and their share in total cost were in case of small, medium, large and at overall level hired human labour (male + female) accounted 19.17 per cent, 11.45 per cent, 8.34 per cent and 10.02 per cent respectively. This human labour used in various farm operations like ploughing, harrowing, sowing, hoeing, weeding, harvesting etc. Bullock labour accounted in case of small, medium, large and at overall level 8.47 per cent, 8.94 per cent, 3.27 per cent and 5.03 per cent respectively. Bullock labour used in the farm operation like ploughing, harrowing, hoeing and transport the farm produce from field to farmhouse. Machine used in farm Operation like ploughing, harrowing, threshing etc. It included the cost in small, medium, large and at overall level were Rs.1400, Rs.2733.33, Rs.3500 and Rs.1940 respectively. Seed was used in case of small, medium, large and overall level Rs.7360, Rs.7220, Rs.7336 and Rs.7336.83 respectively. Manure and fertilizers used in case of small, medium, large and at overall level was13.31 per cent, 
10.55 per cent, 4.27 per cent and 7.88 per cent respectively. An irrigation charge includes electricity bill only and labour charges of irrigation included in hired human charges and family labour charges. In case of small, medium, large and at overall level higher than the non-beneficiary farmers. In nonbeneficiary group costs were lower as compared to beneficiary farmers as there was low level of input used.

\section{Per hectare cost and returns from soybean}

The per hectare cost and return structure of agricultural production, helps the farmer in mapping adjustment in the organization and thereby secure the optimum level of production and income. It is revealed from the Table 3 that at in case of beneficiary overall level average gross return worked out to Rs. 94869.67 The net return obtain at various costs were Rs.31557. at cost 'A', Rs.43587.13 at cost 'B', and Rs. 89555.54 at cost 'C'. The highest input-output ratio at cost ' $C$ ' was recorded in large size group i.e. 1.65 and lowest input-output ratio at cost ' $\mathrm{C}$ ' was recorded in small size group i.e.1.42 At overall level the input-output ratio at cost ' $\mathrm{C}$ ' was 1.65 and medium size group input-output ratio was 1.38 respectively.

In case of beneficiary farmers at small, medium, large and overall level per hectare yield of main produce obtained from soybean 19.24 qt., 22.12 qt., 24.43 qt. and 21.98 qt. respectively it was more as compared to nonbeneficiary farmers which has seen in table 3 . Yield increases 4-5 qt. more than the nonbeneficiary farmers here impact of farm pond shown by providing irrigation to crop in dry spell.

Table.1 Impact on input utilization pattern of beneficiary and non-beneficiary farmers of selected farm ponds in soybean crop (Numbers /ha)

\begin{tabular}{|c|c|c|c|c|c|c|c|c|c|c|}
\hline \multirow{3}{*}{ Sr.No. } & \multirow{3}{*}{\multicolumn{2}{|c|}{ Particular }} & \multicolumn{8}{|c|}{ Land holding size } \\
\hline & & & \multicolumn{2}{|c|}{ Small } & \multicolumn{2}{|c|}{ Medium } & \multicolumn{2}{|c|}{ Large } & \multicolumn{2}{|c|}{ Overall } \\
\hline & & & B & NB & B & NB & B & NB & B & NB \\
\hline 1 & \multicolumn{10}{|c|}{ Hired human labour } \\
\hline $\mathbf{A}$ & Male & Days & 22.80 & 22.7 & 15.07 & 14.62 & 19.66 & 16.11 & 20.04 & 19.66 \\
\hline B & Female & & 25.00 & 24.00 & 22.26 & 16.25 & 25.26 & 18.24 & 16.96 & 18.22 \\
\hline 2 & Bullock labour & Days & 7.80 & 6.10 & 8.60 & 5.12 & 7.00 & 6.00 & 4.73 & 5.78 \\
\hline 3 & Machinery & Hrs. & 5.00 & 4.50 & 5.26 & 5.00 & 5.00 & 4.93 & 3.92 & 5.62 \\
\hline 4 & Seed & $\mathrm{Kg}$ & 73.60 & 58.90 & 73.73 & 70.90 & 118.24 & 71.6 & 82.74 & 76.5 \\
\hline 5 & Manure & Qtl. & 3.10 & 3.36 & 2.26 & 2.40 & 6.36 & 3.93 & 4.92 & 4.63 \\
\hline 6 & \multicolumn{10}{|c|}{ Fertilizer } \\
\hline $\mathbf{A}$ & $\mathrm{N}$ & $\mathrm{Kg}$. & 55.90 & 54.90 & 58.26 & 61.9 & 100.40 & 59.26 & 68.84 & 101.15 \\
\hline B & $\mathrm{P}$ & $\mathrm{Kg}$. & 119.80 & 118.20 & 117.60 & 116.2 & 205.04 & 114.1 & 140.26 & 138.69 \\
\hline 7 & \multicolumn{10}{|c|}{ Family labour } \\
\hline $\mathbf{A}$ & Male & Days & 8.90 & 7.70 & 9.88 & 8.12 & 7.14 & 6.75 & 9.68 & 8.50 \\
\hline $\mathbf{B}$ & Female & & 10.79 & 9.00 & 15.42 & 11.25 & 15.55 & 14.68 & 10.25 & 9.97 \\
\hline
\end{tabular}


Table.2 Impact of per hectare cost of cultivation of soybean of beneficiary and non-beneficiary farmers of selected farm pond (Rs./ha)

\begin{tabular}{|c|c|c|c|c|c|c|c|c|c|}
\hline \multirow{3}{*}{$\begin{array}{l}\text { Sr. } \\
\text { No. }\end{array}$} & \multirow[t]{3}{*}{ Input } & \multicolumn{8}{|c|}{ Size of holding } \\
\hline & & \multicolumn{2}{|c|}{ Small } & \multicolumn{2}{|c|}{ Medium } & \multicolumn{2}{|c|}{ Large } & \multicolumn{2}{|c|}{ Overall } \\
\hline & & B & NB & B & NB & B & NB & B & NB \\
\hline 1 & $\begin{array}{l}\text { Hired human } \\
\text { labour }\end{array}$ & & & & & & & & \\
\hline $\mathbf{a}$ & Male & $\begin{array}{c}4560.00 \\
(8.25)\end{array}$ & $\begin{array}{c}4120.00 \\
(8.89)\end{array}$ & $\begin{array}{c}2653.00 \\
(4.56)\end{array}$ & $\begin{array}{c}2640.00 \\
(4.67)\end{array}$ & $\begin{array}{c}6816.66 \\
(2.76)\end{array}$ & $\begin{array}{c}3144.00 \\
(3.17)\end{array}$ & $\begin{array}{c}3412.00 \\
(3.80)\end{array}$ & $\begin{array}{c}3188.00 \\
(4.21)\end{array}$ \\
\hline $\mathbf{b}$ & Female & $\begin{array}{c}6030.00 \\
(10.92)\end{array}$ & $\begin{array}{c}5634.00 \\
(12.16)\end{array}$ & $\begin{array}{c}4008.00 \\
(6.89)\end{array}$ & $\begin{array}{c}3350.00 \\
(5.93)\end{array}$ & $\begin{array}{c}12645.00 \\
(5.58)\end{array}$ & $\begin{array}{c}5406.00 \\
(5.45)\end{array}$ & $\begin{array}{c}5572.80 \\
(6.22)\end{array}$ & $\begin{array}{c}4834.80 \\
(6.38)\end{array}$ \\
\hline 2 & Bullock labour & $\begin{array}{c}4680.00 \\
(8.47)\end{array}$ & $\begin{array}{c}5340.00 \\
(11.53)\end{array}$ & $\begin{array}{c}5200.00 \\
(8.94)\end{array}$ & $\begin{array}{c}3880.00 \\
(6.86)\end{array}$ & $\begin{array}{c}8075.00 \\
(3.27)\end{array}$ & $\begin{array}{c}4320.00 \\
(4.36)\end{array}$ & $\begin{array}{c}4512.00 \\
(5.03)\end{array}$ & $\begin{array}{c}4392.00 \\
(5.80)\end{array}$ \\
\hline 3 & Machinery & $\begin{array}{c}1400.00 \\
(2.53)\end{array}$ & $\begin{array}{c}1400.00 \\
(3.02)\end{array}$ & $\begin{array}{c}2733.33 \\
(7.70)\end{array}$ & $\begin{array}{c}1250.00 \\
(2.21)\end{array}$ & $\begin{array}{c}3500.00 \\
(1.41)\end{array}$ & $\begin{array}{c}1340.00 \\
(1.35)\end{array}$ & $\begin{array}{c}1970.00 \\
(2.19)\end{array}$ & $\begin{array}{c}1940.00 \\
(2.56)\end{array}$ \\
\hline 4 & Seed & $\begin{array}{c}7360.00 \\
(13.32)\end{array}$ & $\begin{array}{c}7330.00 \\
(15.82)\end{array}$ & $\begin{array}{l}7220.00 \\
(12.42)\end{array}$ & $\begin{array}{c}7213.33 \\
(12.77)\end{array}$ & $\begin{array}{c}7336.83 \\
(5.94)\end{array}$ & $\begin{array}{c}7268.00 \\
(7.33)\end{array}$ & $\begin{array}{c}7306.00 \\
(8.15)\end{array}$ & $\begin{array}{c}7264.00 \\
(9.59)\end{array}$ \\
\hline 5 & Manures & $\begin{array}{c}2500.00 \\
(4.42)\end{array}$ & $\begin{array}{c}2500.00 \\
(5.39)\end{array}$ & $\begin{array}{c}2500.00 \\
(4.30)\end{array}$ & $\begin{array}{c}2500.00 \\
(4.42)\end{array}$ & $\begin{array}{c}2500.00 \\
(4.422)\end{array}$ & $\begin{array}{c}2500.00 \\
(4.30)\end{array}$ & $\begin{array}{l}25000 \\
(3.27)\end{array}$ & $\begin{array}{c}2500.00 \\
(3.30)\end{array}$ \\
\hline 6 & Fertilizer & & & & & & & & \\
\hline $\mathbf{a}$ & $\mathrm{N}$ & $\begin{array}{l}350.00 \\
(0.63)\end{array}$ & $\begin{array}{c}365.00 \\
(0.78)\end{array}$ & $\begin{array}{l}350.00 \\
(0.60)\end{array}$ & $\begin{array}{c}352.00 \\
(0.62)\end{array}$ & $\begin{array}{l}717.91 \\
(0.29)\end{array}$ & $\begin{array}{l}358.80 \\
(0.36)\end{array}$ & $\begin{array}{l}354.40 \\
(0.39)\end{array}$ & $\begin{array}{c}365.00 \\
(0.47)\end{array}$ \\
\hline $\mathbf{b}$ & $\mathrm{P}$ & $\begin{array}{c}2357.76 \\
(4.26)\end{array}$ & $\begin{array}{c}2700.00 \\
(5.83)\end{array}$ & $\begin{array}{c}3286.66 \\
(5.65)\end{array}$ & $\begin{array}{c}2613.33 \\
(4.62)\end{array}$ & $\begin{array}{c}5329.16 \\
(2.16)\end{array}$ & $\begin{array}{c}2832.00 \\
(2.85)\end{array}$ & $\begin{array}{c}2891.04 \\
(3.22)\end{array}$ & $\begin{array}{c}2740.00 \\
(3.61)\end{array}$ \\
\hline 7 & Irrigation & $\begin{array}{l}447.70 \\
(0.81)\end{array}$ & $\begin{array}{c}563.00 \\
(1.21)\end{array}$ & $\begin{array}{l}256.00 \\
(0.44)\end{array}$ & $\begin{array}{l}124.73 \\
(0.22)\end{array}$ & $\begin{array}{l}568.75 \\
(0.23)\end{array}$ & $\begin{array}{l}97.16 \\
(0.09)\end{array}$ & $\begin{array}{l}308.14 \\
(0.34)\end{array}$ & $\begin{array}{l}198.00 \\
(0.26)\end{array}$ \\
\hline 8 & Plant protection & $\begin{array}{c}3280.00 \\
(5.94)\end{array}$ & $\begin{array}{c}2590.00 \\
(5.59)\end{array}$ & $\begin{array}{c}2293.33 \\
(3.94)\end{array}$ & $\begin{array}{c}2286.00 \\
(4.04)\end{array}$ & $\begin{array}{c}4791.66 \\
(1.94)\end{array}$ & $\begin{array}{c}1772.40 \\
(1.78)\end{array}$ & $\begin{array}{c}2536.00 \\
(2.83)\end{array}$ & $\begin{array}{c}2090.00 \\
(2.76)\end{array}$ \\
\hline 9 & Depreciation & $\begin{array}{c}2170.00 \\
(3.92)\end{array}$ & $\begin{array}{c}1466.00 \\
(3.16)\end{array}$ & $\begin{array}{c}1640.00 \\
(2.82)\end{array}$ & $\begin{array}{c}1237.33 \\
(2.19)\end{array}$ & $\begin{array}{c}3672.08 \\
(1.48)\end{array}$ & $\begin{array}{c}1450.40 \\
(1.46)\end{array}$ & $\begin{array}{l}1843.8 \\
(2.05)\end{array}$ & $\begin{array}{c}1389.00 \\
(1.83)\end{array}$ \\
\hline 10 & Threshing & $\begin{array}{c}2730.00 \\
(4.94)\end{array}$ & $\begin{array}{c}2410.00 \\
(5.20)\end{array}$ & $\begin{array}{c}2760.00 \\
(4.74)\end{array}$ & $\begin{array}{c}1922.06 \\
(3.40)\end{array}$ & $\begin{array}{c}5445.83 \\
(2.20)\end{array}$ & $\begin{array}{c}2236.16 \\
(2.25)\end{array}$ & $\begin{array}{c}2732.00 \\
(3.05)\end{array}$ & $\begin{array}{c}2176.00 \\
(2.87)\end{array}$ \\
\hline 11 & Transportation & $\begin{array}{l}257.00 \\
(0.50)\end{array}$ & $\begin{array}{l}234.00 \\
(0.50)\end{array}$ & $\begin{array}{l}274.00 \\
(0.47)\end{array}$ & $\begin{array}{l}166.26 \\
(0.29)\end{array}$ & $\begin{array}{l}482.91 \\
(0.19)\end{array}$ & $\begin{array}{l}396.00 \\
(0.39)\end{array}$ & $\begin{array}{l}258.00 \\
(0.28)\end{array}$ & $\begin{array}{c}294.00 \\
(0.38)\end{array}$ \\
\hline 12 & Land Revenue & $\begin{array}{l}176.00 \\
(0.31)\end{array}$ & $\begin{array}{l}134.00 \\
(0.28)\end{array}$ & $\begin{array}{l}190.00 \\
(0.320\end{array}$ & $\begin{array}{l}145.33 \\
(0.25)\end{array}$ & $\begin{array}{l}805.00 \\
(0.32)\end{array}$ & $\begin{array}{c}275.92 \\
(0.27)\end{array}$ & $\begin{array}{l}281.00 \\
(0.31)\end{array}$ & $\begin{array}{l}142.00 \\
(0.18)\end{array}$ \\
\hline 13 & $\begin{array}{l}\text { Interest. on } \\
\text { working } \\
\text { capital@ 6\% } \\
\text { annum }\end{array}$ & $\begin{array}{c}1710.00 \\
(3.09)\end{array}$ & $\begin{array}{l}143.00 \\
(3.09)\end{array}$ & $\begin{array}{c}1455.33 \\
(2.50)\end{array}$ & $\begin{array}{c}1422.667 \\
(2.51)\end{array}$ & $\begin{array}{c}2863.75 \\
(1.16)\end{array}$ & $\begin{array}{c}1257.60 \\
(1.27)\end{array}$ & $\begin{array}{c}1494.20 \\
(1.66)\end{array}$ & $\begin{array}{c}1341.00 \\
(1.77)\end{array}$ \\
\hline 14 & COST A & $\begin{array}{c}44471.45 \\
(80.53)\end{array}$ & $\begin{array}{c}39608.25 \\
(85.53)\end{array}$ & $\begin{array}{c}37134 \\
(63.90)\end{array}$ & $\begin{array}{c}31473.68 \\
(55.72)\end{array}$ & $\begin{array}{c}69350.16 \\
(28.11)\end{array}$ & $\begin{array}{c}32282.04 \\
(32.60)\end{array}$ & $\begin{array}{c}37374.00 \\
(41.73)\end{array}$ & $\begin{array}{c}33504.77 \\
(44.25)\end{array}$ \\
\hline 15 & $\begin{array}{l}\text { interest on fixed } \\
\text { capital @ } 10 \% \\
\text { annum }\end{array}$ & $\begin{array}{c}2165.00 \\
(3.92)\end{array}$ & $\begin{array}{l}1433.5 \\
(3.09)\end{array}$ & $\begin{array}{c}2132.667 \\
(3.67)\end{array}$ & $\begin{array}{c}2153.333 \\
(3.81)\end{array}$ & $\begin{array}{c}4284.583 \\
(1.73)\end{array}$ & $\begin{array}{c}2141.60 \\
(2.16)\end{array}$ & $\begin{array}{c}2143.60 \\
(2.39)\end{array}$ & $\begin{array}{l}2003.50 \\
(2.64)\end{array}$ \\
\hline 16 & $\begin{array}{l}\text { Rental value of } \\
\text { land }\end{array}$ & $\begin{array}{c}13097.08 \\
(23.71)\end{array}$ & $\begin{array}{c}9946.66 \\
(21.47)\end{array}$ & $\begin{array}{c}15256.39 \\
(26.25)\end{array}$ & $\begin{array}{c}12815.00 \\
(22.68)\end{array}$ & $\begin{array}{c}68815.97 \\
(27.89)\end{array}$ & $\begin{array}{c}24396.33 \\
(24.63)\end{array}$ & $\begin{array}{c}24211.75 \\
(27.03)\end{array}$ & $\begin{array}{c}18032.00 \\
(23.81)\end{array}$ \\
\hline 16 & COST B & $\begin{array}{r}63108.95 \\
(114.29)\end{array}$ & $\begin{array}{c}53833.46 \\
(116.25)\end{array}$ & $\begin{array}{l}54624 \\
(94.00)\end{array}$ & $\begin{array}{c}46791 \\
(82.84)\end{array}$ & $\begin{array}{l}103355 \\
(41.89)\end{array}$ & $\begin{array}{c}46414.08 \\
(46.87)\end{array}$ & $\begin{array}{c}54850 \\
(61.24)\end{array}$ & $\begin{array}{c}48011.18 \\
(63.40)\end{array}$ \\
\hline 17 & Family Labour & & & & & & & & \\
\hline $\mathbf{a}$ & Male & $\begin{array}{c}3350.00 \\
(4.26)\end{array}$ & $\begin{array}{c}2400.00 \\
(5.18)\end{array}$ & $\begin{array}{c}1950.00 \\
(3.35)\end{array}$ & $\begin{array}{c}6297.22 \\
(11.14)\end{array}$ & $\begin{array}{c}6433.00 \\
(2.60)\end{array}$ & $\begin{array}{c}3708.66 \\
(3.74)\end{array}$ & $\begin{array}{c}2675.83 \\
(2.98)\end{array}$ & $\begin{array}{c}4223.00 \\
(5.57)\end{array}$ \\
\hline b & Female & $\begin{array}{c}1944.00 \\
(3.52)\end{array}$ & $\begin{array}{l}1980.00 \\
(4.87)\end{array}$ & $\begin{array}{c}2137.00 \\
(3.68)\end{array}$ & $\begin{array}{c}6127.50 \\
(10.84)\end{array}$ & $\begin{array}{c}6255.93 \\
(2.53)\end{array}$ & $\begin{array}{c}3257.20 \\
(3.28)\end{array}$ & $\begin{array}{c}2612.77 \\
(2.91)\end{array}$ & $\begin{array}{c}3862.85 \\
(5.10)\end{array}$ \\
\hline 18 & Cost $\mathrm{C}$ & $\begin{array}{c}55217.24 \\
(100)\end{array}$ & $\begin{array}{c}46306.77 \\
(99.99)\end{array}$ & $\begin{array}{c}58106.79 \\
(100)\end{array}$ & $\begin{array}{l}56483 \\
(100)\end{array}$ & $\begin{array}{c}246691.6 \\
(99.99)\end{array}$ & $\begin{array}{c}99021.15 \\
(100)\end{array}$ & $\begin{array}{c}89555.54 \\
(100)\end{array}$ & $\begin{array}{c}75716.89 \\
(100)\end{array}$ \\
\hline
\end{tabular}

(Figure in parentheses indicates the percentages to total cost C) 
Table.3 Impact of per hectare cost and returns on soybean beneficiary and non-beneficiary farmers of selected farm ponds

\begin{tabular}{|c|c|c|c|c|c|c|c|c|c|}
\hline \multirow{3}{*}{$\begin{array}{c}\text { Sr. } \\
\text { No } \\
1\end{array}$} & \multirow{3}{*}{$\begin{array}{c}\text { Particulars } \\
\text { Yield }\end{array}$} & \multicolumn{8}{|c|}{ Size of holding } \\
\hline & & \multicolumn{2}{|c|}{ Small } & \multicolumn{2}{|c|}{ Medium } & \multicolumn{2}{|c|}{ Large } & \multicolumn{2}{|c|}{ Overall } \\
\hline & & B & NB & B & NB & B & NB & B & NB \\
\hline $\mathbf{A}$ & $\begin{array}{c}\text { Main } \\
\text { Produce }(\mathrm{Qtl})\end{array}$ & 19.03 & 14.43 & 22.12 & 18.53 & 24.43 & 35.65 & 21.98 & 26.02 \\
\hline B & $\begin{array}{c}\mathrm{By} \\
\text { produce }(\mathrm{Qtl})\end{array}$ & 9.85 & 7.84 & 12.33 & 11.02 & 23.14 & 16.83 & 17.21 & 13.56 \\
\hline \multirow[t]{3}{*}{2} & $\begin{array}{l}\text { Gross return } \\
\text { (Rs) }\end{array}$ & 78582.50 & 59680.00 & 91538.33 & 76890.00 & 96501.42 & 77002.38 & 94869.67 & 76853.27 \\
\hline & $\begin{array}{c}\text { Value of } \\
\text { main } \\
\text { produce }(\mathrm{Rs})\end{array}$ & 76120.00 & 57780.00 & 88480.00 & 74134.00 & 198400.00 & 142609.50 & 140968.00 & 104800.00 \\
\hline & $\begin{array}{l}\text { Value of by } \\
\text { produce(Rs) }\end{array}$ & 2462.50 & 1960.00 & 3058.33 & 2756.00 & 5785.00 & 4209.50 & 4302.50 & 3392.00 \\
\hline \multirow[t]{4}{*}{3} & Costs (Rs) & & & & & & & & \\
\hline & Cost A & 32280.70 & 31446.10 & 30746.99 & 27101.40 & 31753.00 & 28952.90 & 31557.09 & 28896.10 \\
\hline & Cost B & 44053.32 & 41513.12 & 43188.47 & 36840.59 & 43639.85 & 38856.53 & 43587.13 & 38783.07 \\
\hline & Cost C & 55217.24 & 4636.77 & 58106.79 & 56483.00 & 246691.60 & 99021.15 & 89555.54 & 75716.89 \\
\hline \multirow[t]{4}{*}{4} & $\begin{array}{l}\text { Net Return } \\
\text { Over (Rs.) }\end{array}$ & & & & & & & & \\
\hline & Cost 'A' & 46302.33 & 28233.9 & 60791.34 & 49788.60 & 61363.09 & 44720.34 & 57791.32 & 43099.37 \\
\hline & Cost 'B' & 34529.18 & 18166.88 & 48349.86 & 40049.41 & 44091.93 & 30588.30 & 40181.17 & 28592.96 \\
\hline & Cost ' $C^{\prime}$ & 23365.26 & 55043.27 & 33431.54 & 20407.00 & 37954.98 & 24165.29 & 35168.72 & 21150.42 \\
\hline \multirow[t]{4}{*}{5} & $\begin{array}{c}\text { Input-Output } \\
\text { Ratio at }\end{array}$ & & & & & & & & \\
\hline & Cost ' $\mathrm{A}^{\prime}$ & 2.43 & 1.89 & 2.91 & 2.83 & 2.75 & 2.39 & 2.56 & 2.28 \\
\hline & Cost 'B' & 1.78 & 1.43 & 2.11 & 2.08 & 1.84 & 1.66 & 1.73 & 1.59 \\
\hline & Cost ${ }^{\prime} C^{\prime}$ & 1.42 & 1.02 & 1.57 & 1.36 & 1.65 & 1.46 & 1.59 & 1.38 \\
\hline
\end{tabular}

In conclusion the per hectare input utilization for soybean crop at overall level, the beneficiary farmer per hectare cost of cultivation of soybean for overall level the use of various inputs for soybean on beneficiary farms was slightly higher than non-beneficiary farms. At overall level family labour, fertilizers and manures were used more in case of beneficiary farmers as compared to non-beneficiary farmers. While bullock labour, seed, machinery was more in case non-beneficiary farmers as compared to beneficiary farmers. Use of seed and farm yard manure per hectare did not vary much among the beneficiary and non-beneficiary farms. Use of chemical fertilizers on beneficiary farms was slightly higher than the non-beneficiary farms.
Intergroup comparison of beneficiary and nonbeneficiary farms also revealed the same trend.

The overall per ha. total cost of cultivation i.e. cost ' $\mathrm{C}$ ' ranges from Rs.89555.54 to Rs. 75716.89 for beneficiary and non-beneficiary farmers respectively. For beneficiary farmers, except in large size group it was Rs.246691.6, in medium size group it was Rs.58106.79 and in case of small size group of farmers it was Rs. 55217.24 respectively. The higher total cost on large size farmer was obviously due to higher use of input. The overall per ha. gross return of beneficiary and non-beneficiary farmers was and Rs. 76853.27 respectively. In case of beneficiary farmers at overall level average 
gross return was Rs. 94869.67 While in case of non-beneficiary farmers it was Rs. 76853.27. It means production was more in case of beneficiary farmers as compared to nonbeneficiary farmers. In case of beneficiary farmers at overall level the Output-input ratio at cost ' $\mathrm{C}$ ' was 1.59, while in case of nonbeneficiary farmers it was 1:38. It shows that the beneficiary farmers were more profitable than non-beneficiary farmers.

The impact of farm pond construction on their field increase water level of well and also for their field to provide water during crop season whenever necessary to increase a crop production. Higher regression coefficient and consequently higher factor productivity for beneficiary farms were obviously due to farm pond availability on these farms.

\section{References}

Alshi, M. R., Joshi, C.K. and Khedakar U. J. (1991). Economic evaluation of Gunjwatershed development project in Akola district of Maharashtra. Indian Journal of Agriculture economics. 44 (3): 310-311.

Ambati, R. R. Mujumdar, G. and Reddy, A.R. (2011). Validation of farm pond size for irrigation during drought. Indian Journal of Agronomy. 56 (4): 356-364.

Andure (Yawale) Neeta, Verma A. A., Langevar V. S. and Kadam A. R. (2017). The impact of farm pond on the farms of Amaravati taluka region. International Journal of Scientific Research. 6 (4): 734 737.

Anonymous (2013). Impact of farm ponds on beneficiary farmers of western Viderbha. Research review committee project submitted, on 2nd April 2013 at RRC meeting Dr. Panjabrao Deshmukh Krishi
Vidyapeeth, Akola.

Indian Journal of Agriculture economics. 46 (3): 313

Deshmukh, J. M., Hyalij, V. D., Surdkar., D. D. and Badgire B.B. (2017). Impact assessment of farm ponds on beneficiaries. International Journal of Current Microbiology and Applied Sciences. 6 (9): 1712-1717.

Chavai, A. M., Rakshe U. V. and Shinde S. B. (2015). Impact of farm ponds on beneficiary farmers of Maharashtra. International Journal of tropical Agriculture. 33(4): 3525-3528.

Desai, R., Patil, B. L., Kunnal, L.B., Jayshree H. and Basvaraj H. (2006), Impact assessment of farm-ponds in Dharwad district of Karnataka. Karnataka Journal of Agricultural sciences. 20 (2) 426- 427.

Deshmukh, J. M., Hyalij, V. D., Surdkar., D. D. and Badgire B.B. (2017). Impact assessment of farm ponds on beneficiaries. International Journal of Current Microbiology and Applied Sciences. 6 (9): 1712-1717.

Kulkarni, V. S., Sankarmurthy, H.G. and Kairsur V. R. (1989). Impact on watershed management on dry land farming. A case study of Asundialawartershed in Dharwad district in Karnataka. Indian Journal of Agriculture economics. 44(1): 272.

Sindhu, R. S., Sindhu, J. S. and Singh, S. (1991). A study on economic impact of Kothewadi watershed development programme in Kandi area of Punjab. Indian Journal of Agriculture economics. 46 (3): 312.

Suyavanshi B. N., Patil and Tuse B.P. (1991). A study on economic impact of Kothewadi watershed development programme in Maharashtra. Indian Journal of Agriculture economics. 46 (3): 326-327.

\section{How to cite this article:}

Chakranarayan, A. D., S. C. Nagpure, J. R. Kankate and Patil, R. K. 2020. Cost and Return Analysis of Beneficiaries and Non-Beneficiary Farm Pond Farmers for Soybean Cultivation in Washim District. Int.J.Curr.Microbiol.App.Sci. 9(12): 2259-2265. doi: https://doi.org/10.20546/ijcmas.2020.912.267 